\title{
Running suture hemostatic technique versus standard reconstruction of the surgical bed in Zero - Ischemia Time mini-flank Open Partial Nephrectomies. Retrospective, Match-Paired Case-Control Study
}

\author{
Petar Kavaric, Aleksandar Magdelinic, Marko Vukovic \\ Faculty of Medicine, University of Montenegro, Podgorica, Montenegro.
}

\begin{abstract}
Summary Objective: To estimate the efficacy of our technique of zero ischemia time partial nephrectomy (ZTPN) with hemostatic running suture and compare it to the standard technique, in terms of perioperative complications, operative time (OT) and estimated blood loss (EBL).

Materials and methods: We retrospectively analysed 180 consecutive patients who underwent ZTPN using a supra $11^{\text {th }}$ or supra $12^{\text {th }}$ rib mini flank approach. First group numbered 90 patients treated with running suture hemostatic technique (RSHT), while the control group enrolled 90 patients in whom we performed standard reconstruction technique (SRT). According the propensity score, both groups were similar in terms of tumor size, age and PADUA score. Patients with solitary tumour limited to the kidney (T1-T2a) were included. Our technique included a running suture of surgical bed edges and closure of the renal cortex by the positioning of peri-renal fat within the cortical bed and fixation with interrupted sutures. Results: PADUA score and tumor size were comparable between groups $(7.12 \pm 1.33$ vs $7.1 \pm 2.11, p=0.4$ and $52.9 \pm$ 14.8 vs $50.0 \pm 13.2, p=0.3)$. The mean operative time (OT) was significantly longer in first group (165.2 vs 95, $p=0.04)$, while median estimated blood loss (EBL) was significantly reduced (250 vs $460 \mathrm{ml}, p=0.02$ ). Surgical resection margins were negative in $100 \%$ of cases and no patient developed a local or distant recurrence during follow up. There was signifcant difference in postoperative GFR value between groups $(p<0.05)$.

Conclusions: Our technique could be safely performed in local, low volume facilities, thus reducing the need for expensive and more challenging minimal invasive surgical techniques..
\end{abstract}

KEY WORDS: Nephrectomy; Ischemia time; Hemostatic technique.

Submitted 12 February 2019; Accepted 13 February 2019

\section{INTRODUCTION}

Partial nephrectomy (PN) for localized kidney tumor has oncological outcomes similar to that of radical surgery (1). According to current guidelines, patients with low grade renal cell carcinoma (RCC) should undergo nephron-sparing surgery rather than radical nephrectomy whenever possible (EAU guidelines). Utilizing minimally invasive surgical approaches to open nephrectomy, two mini-flank open techniques have been devel- oped - the supra $11^{\text {th }}$ rib mini flank approach and supra $12^{\text {th }}$ rib approach $(2,3)$. These techniques provide optimum anatomical exposure and better aesthetic outcomes with a low risk of long-term complications (2). Typically, for a lower pole tumor, a supra $12^{\text {th }}$ rib incision is more appropriate, while for mid and upper pole tumors a supra $11^{\text {th }}$ incision ispreferred (3). Clamping of the hilar vessels during partial nephrectomy may cause ischemic damage to the kidney and subsequent chronic renal impairment, which implies the necessity for improving zero-ischemia time techniques, especially for long lasting procedures, where ischemia time can be more than 25-30 minutes $(4,5)$. This led to the development of techniques such as zero ischemia time partial nephrectomy (ZTPN) (6). There are however difficulties with this approach, which include increased intraoperative blood loss compared with on-clamp procedure, hence requiring new, technically less demanding haemostatic techniques with comparable blood loss.

The aim of this study was to present our approach open ZTPN using a running suture hemostatic technique for surgical bed - in order to reduce intraoperative blood loss and maintain stable renal function (RF), comparing to standard reconstruction technique.

\section{Patients And methods}

From 543 patients who underwent tumour nephrectomy in our clinic between January 1997 and March 2017, we retrospectively analysed 186 consecutive patients who underwent PN using supra $11^{\text {th }}$ or supra $12^{\text {th }}$ rib mini flank approach $(2,3)$. First group numbered 96 patients with running suture hemostatic technique (RSHT), while the control group enrolled 90 patients in whom we performed standard reconstruction technique (SRT).

The patients who underwent RSHT were matched to control group according to the following variables: tumor size, age and anatomic classification of renal tumors (PADUA) score. Finally, 90 patients from the first group were matched to 90 patients from the control group. The indication for surgery was solitary renal tumor limited to the kidney (cT1-cT2a). We used preoperative CT or MRI tumor staging according to the 
2004 World health organization (WHO) classification of renal epithelial tumors (7). Patients with the following criteria were excluded: those with blood disorders; evidence of locally advanced or systemic disease; regional adenopathy or previous kidney surgery. The presence of hereditary renal cancers was no contraindication for surgery. The variables we examined when reviewing our database were demographics (age, gender, body mass index), lesion characteristics (location, centrality and size), pathological stage and histological subtype, perioperative variables operative time (OT), estimated blood loss (EBL), postoperative glomerular filtration rate (pGFR), length of hospital stay and intraoperative and postoperative complications (POC) (3). POC were classified according to the modified Clavian system (8).

- The primary end points evaluated included EBL, postoperative creatinine and pGFR, POC and hospital stay.

- The secondary end points evaluated included OT, transfusion rate and surgical margin status.

Tumour histology was evaluated using modified Heidelberg histopathological classifications of renal tumors (9).

\section{Surgical approach and hemostatic technique}

After positioning a patient in a standard flank position, we then perform a supra $11^{\text {th }}$ or supra $12^{\text {th }}$ skin and subcutaneous incision, using the mini-flank technique as described by Diblasio et al. (2). After the transection of abdominal wall muscle layers and division of the transverses abdominis fibers, we use a combination of blunt and sharp dissection (finger and Metzenbaum scissors) to divide the transversalis and lumbodorsal fascia, with displacement of the pleura using a sponge stick. We use a self-retaining retractor with an additional bladder blade or Morris retractor to retract the $10^{\text {th }}$ or $11^{\text {th }}$ rib superiorly. Typically, we do not perform resection of the $12^{\text {th }}$ rib. After accessing the retroperitoneal space using bluntdissection and reflecting the kidney with surrounding fat tissue medially, we create the plane between the

A.

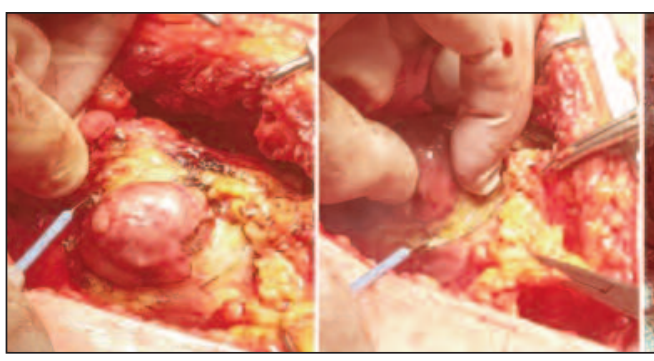

D.

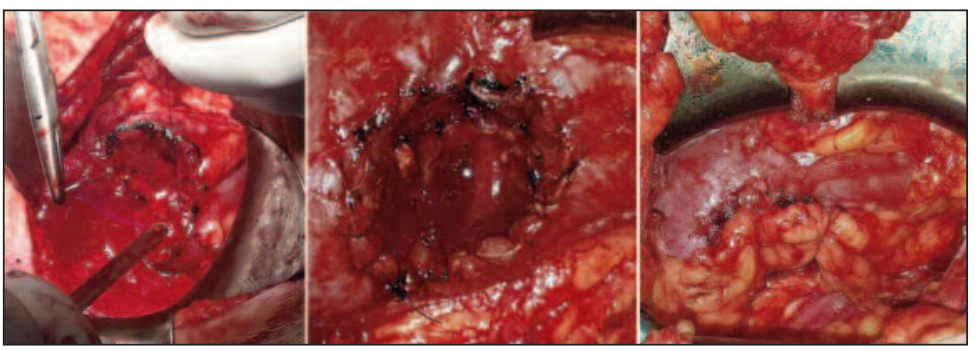

quadrates lumborum and psoas muscle. In the case of upper pole tumours, after medial and lateral mobilisation of the kidney we isolate the ureter from the lower pole and place it in a yellow vessel loop. When operating upper pole tumors, the adrenal gland would be inspected $\&$ palpated, and if there is no indication for adrenalectomy we proceed with mobilization of the upper pole of the kidney using LigaSure bipolar current (LigaSuretm, Covidien, Minneapolis, USA). After mobilization of surrounding fat, the kidney is carefully inspected to determine the depth and proximity of the tumour to the renal vessels and collecting system (2). For centrally located tumors and for endophitic ones, we use intraoperative ultrasonography in order to accurately identify tumour borders. Upon demarcation of tumor contours with monopolar current (Figure 1A), we use sharp dissection of tumour tissue together with resection of an approximately $0.5 \mathrm{~cm}$ thick rim of tissue from the tumour bed (Figure 1B-C). We do not perform tumour margins frozen section routinely, even for deep renal tumour specimen. Prominent arterial branches within the tumor bed are ligated with 2/0 Vicryl ligature or clipped using surgical microclips, in order to selectively devascularize the tumour without interruption of normal renal perfusion (6). With incidental break (what do you mean) within the collecting system, the calyces are sutured with 4/0 PDS suture (Figure 1D). After the excision of tumour tissue, we utilize running 4/0 PDS suture of surgical bed edges, with additional hemostatic sutures in case of minor bleeding within the surgical bed (Figure $1 \mathrm{E})$. At the end, we close the renal cortex by placing perirenal fat within the cortical bed and placing size 0 chromic liver interrupted sutures (Figire 1F).

Standard reconstruction technique consists of tumor resection and reconstruction of the surgical bed with single, interrupted sutures, followed by application of hemostatic agents within resection cavity (Surgicel; Johnson and Johnson, New Brunswick, New Jersey). The renal capsule is reaproximated using 0 - Vicryl sutures pledged with Surgicel to prevent tearing of the renal cortical capsule and further bleeding (10). The surgical incision is closed using 3/0 absorbable sutures in a subcuticular fashion (2). During the early postoperative period (48h), blood pressure is tightly controlled (e.g. mean arterial pressure (MAP) between $60-100 \mathrm{mmHg}$ ) in order to avoid additional bleeding from renal parenchyma, but also to maintain safe tissue perfusion and oxygenation, preserving normal postoperative RF.

Figure 1.

Surgical technique of ZTPN using running suture technique for surgical bed with fat tissue tamponade: demarcation of tumor with monopolar current (A); sharp dissection of tumor tissue with approximately $0.5 \mathrm{~cm}$ thick rim of renal tissue (B); tumor appearance after $\mathrm{C}$ ); suturing of ruptured calyces and tumor bed (D); hemostatic running suture of wound edges $(E)$; Fat tamponade (F). 


\section{Statistical analysis}

For statistical analysis we used SPPS v16.0, SPPS, Chicago, IL, USA. Methods of statistical description and significance included the Student $\mathrm{T}$ test and Mann Whitney U test. Descriptive analyses were also generated and some data are reported as median, interquartile range (IQR), or number (\%). The difference of the obtained values was considered to be significant when $\mathrm{p}<0.05$, and highly significant when $\mathrm{p}<0.01$.

\section{RESULTS}

Following exclusion criteria and score matching, 180 patients were eligible for this study. According the propensity score, both groups were similar in terms of tumor size, age and PADUA score $(52.9 \pm 14.8$ vs $50.0 \pm$ $13.2 \mathrm{~mm}, \mathrm{p}=0.3 ; 57 \pm 17.26$ vs $55 \pm 12.19$ years, $\mathrm{p}=0.5$ and $7.12 \pm 1.33$ vs $7.1 \pm 2.11, \mathrm{p}=0.4$, respectively) (Table 1). Median follow up time was 52.75 months in first group and 59.25 within control group ( $\mathrm{p}=0.3$ ). Additionally, hospital stay and surgical margin status did not differ between the groups. The mean PADUA score in first group was $7.12 \pm 1.33$ where $14.7 \%$ of patients had a score $>8$ and 5 patients $(5.25 \%$ ) had a score $>10$.

The majority of masses $(55.5 \%)$ were malignant with predominance of the clear cell subtype (67.7\%) and exophitic growth (78.9\%). The demographic data are given in Table 2. Intraoperatively, three patients (3.3\%) from the first group and 7 from the control group $(7.77 \%)$ required radical nephrectomy due to hilar or deeply penetrating endophytic tumours. Concomitant radical tumor nephrectomy of the other kidney was required in 9 and 7 patients (10\% vs $7.77 \%$ ), with no clinical confirmation of hereditary cancer occurence.

No other complications were recorded during surgery. There were $11.2 \%$ and $23.3 \%$ POC during follow-up, which were predominantly Clavien grade II (Table 1).

Table 1.

Comparison of perioperative outcomes between two groups.

\begin{tabular}{|lcc|}
\hline \multicolumn{3}{|c|}{ Mean (SD)/Median (IQR) } \\
\hline $\mathbf{N}$ & $\begin{array}{c}\text { Group I } \\
\mathbf{9 0} \text { patients }\end{array}$ & $\begin{array}{c}\text { Control group } \\
\mathbf{9 0} \text { patients }\end{array}$ \\
\hline Age (years) & $57(17.26)$ & $55(12.19)$ \\
\hline PADUA score & $7.12(1.33)$ & $7.1(2.11)$ \\
\hline Tumor size (mm) & $52.9(14.8)$ & $50(13.2)$ \\
\hline Operative time & $165.2(47.31)$ & $\mathbf{9 5 ( 3 2 . 1 )}{ }^{*}$ \\
\hline Estimated blood loss (ml) & $250(100-350)$ & $\mathbf{4 6 0 ~ ( 1 7 0 - 5 3 0 )}$ \\
\hline Hospital stay (days) & $5(2.5)$ & $7(1.5)$ \\
\hline Surgical margin & Number (\%) & \\
\hline Transfusion rate & $0(100)$ & $0(100)$ \\
\hline Complications & $1(1.1)$ & $2(2.2)$ \\
\hline Clavien I & 11 (12.22) & $\mathbf{2 1 ~ ( 2 3 . 3 )}$ \\
\hline Clavien II & 3 & 7 \\
\hline Clavien III & 5 & 8 \\
\hline Clavien IV & 2 & 2 \\
\hline * Statistically significant difference between corresponding groups $(\mathbf{p}<0.05)$. \\
\hline
\end{tabular}

Table 2.

Demographic data within first group.

\begin{tabular}{|lc|}
\hline \multicolumn{2}{|c|}{ Mean (SD)/Median (IQR) } \\
\hline $\mathbf{N}$ & $\mathbf{9 0}$ patients \\
\hline Male, $\mathrm{n}(\%)$ & $60(66.6)$ \\
\hline Female, $\mathrm{n}(\%)$ & $30(33.3)$ \\
\hline Median body mass index, $\mathrm{kg} / \mathrm{m}^{2}$ & $30.75(7.45)$ \\
\hline ASA class & $3(1-4)$ \\
\hline Indication for PN & Number (\%) \\
Elective & $80(88.2)$ \\
Solitary kidney & $4(4.4)$ \\
Bilateral tumors & $6(6.6)$ \\
\hline Tumor location & Number (\%) \\
Upper pole & $45(50)$ \\
Mid pole & $9(10)$ \\
Lower pole & $31(34.4)$ \\
Renal hilus & $5(5.6)$ \\
\hline Tumor histology & Number (\%) \\
Clear cell & $61(67.7)$ \\
Papillary & $15(16.7)$ \\
Oncocytoma & $7(7.8)$ \\
Chromophobe & $3(3.4)$ \\
Multilocular cystic & $4(4.4)$ \\
\hline
\end{tabular}

Table 3.

Comparison of several parameters before and after surgical treatment between groups.

\begin{tabular}{|c|c|c|}
\hline \multicolumn{3}{|c|}{ Mean (SD)/Median (IQR) } \\
\hline First group & Before treatment & $72 \mathrm{~h}$ after the treatment \\
\hline Creatinine (mg/dl) & $1.16(0.5)$ & $1.10(0.46)$ \\
\hline$\overline{\mathrm{GFR}}(\mathrm{ml} / \mathrm{min})$ & $91.66(9.5)$ & $95.86(8.4)^{* *}$ \\
\hline Control group & Before treatment & $72 \mathrm{~h}$ after the treatment \\
\hline Creatinine (mg/dl) & $1.10(0.3)$ & $1.55(0.76)$ \\
\hline$\overline{G F R}(\mathrm{ml} / \mathrm{min})$ & $88.25(8.7)$ & $77(6.8)$ \\
\hline \multicolumn{3}{|c|}{$\begin{array}{l}\text { * Statistically significant difference comparing preoperativeand postoperative values } \\
\text { within first group }(p<0.05) \text {. } \\
\text { ** Statistically significant difference comparing postoperative values between first and } \\
\text { control group }(p<0.05) \text {. }\end{array}$} \\
\hline
\end{tabular}

Postoperative transfusion rate was $1.1 \%$ and $2.2 \%$ with a maximum of 1 blood unit required; mean EBL was 250 and $460 \mathrm{ml}$, while average OT was 165.2 and $95 \mathrm{~min}$. Intraoperative ultrasound was used in 26 patients (28.8\%). Surgical resection margins were negative in $100 \%$ of all cases (Table 1) and no patient developed a local or distant recurrence during follow up. Table 3 shows pre and postoperative parameters between groups. There was no significant difference in preoperative creatinine value, GFR or haemoglobin (Hgb) between groups $(p=0.43 ; p=0.51$ and $p=0.6)$. Nevertheless, GFR was significantly increased in first group during the early postoperative period $(98.86 \pm 8.4$ vs $77 \pm 6.8, \mathrm{p}=0.01)$.

\section{Discussion}

The primary goal of this study was to determine whether RSHT could substantially decrease the morbidity associated with SRT, regarding EBL, postoperative creatinine and pGFR. Our results showed that EBL and POC were sig- 
nificantly reduced within first group, while postoperative GFR increased, compared to control group of patients. Nevertheless, OT was significantly prolonged using RSHT. The main goal in PN is to achieve negative tumor margins with a minimal decrease in renal function $(\mathrm{RF})$ and minimal blood loss. Since warm ischemia may be detrimental to RF and cold ischemia may be difficult to achieve during minimally invasive PN, several techniques have been developed in order to avoid clamping of the renal artery (6). Anatomical zero-ischemia PN, introduced by Gill et al. (11) was based on clipping of tumor-specific arterial branches, in order to devascularize the tumor without interruption of normal renal perfusion. This technique led to low EBL (206 ml) with $100 \%$ negative surgical margins and a transfusion rate of $21 \%$ with POC grade $>3$ of $3.5 \%$. We used a similar concept during excision of tumour tissue and after final hemostasis of the surgical bed, mean EBL was $250 \mathrm{ml}$ in first and $460 \mathrm{ml}$ in control group, with a transfusion rate of $1.1 \%$ and $2.2 \%$ and a $100 \%$ negative surgical margin. Although we performed open PN in both groups, mean EBL was significantly lower in group treated with RSHT, which could be associated with meticulous surgical technique and suturing of surgical bed edges with additional fat tissue tamponade. The importance of the improving outcomes of RF through technical modification of resection techniques has already been emphasized (12), with an emphasis on minimization of resection margins and amount of tissue incorporated into renorrhaphy. Desai et al. (13) shares our attitudes on 'tissue-sparing' technique using running suture for wound edges through their reporting of point-specific hemostasis of the parenchymal defect. However, it is worth noting limitations in comparing these techniques with simple interrupted renorrhaphy, as our study describes open technique of PN, while majority of other studies outline a laparoscopic or robotic assisted minimal invasive approach.

One of the few papers comparing perioperative and functional outcomes for patients treated with open, off-clamp PN, is research made by Smith et al. (14), where authors retrospectively evaluated 192 patients and reported long operative times (226.5 min), significant EBL (500 ml) and a high transfusion rate $(42 \%)$. Our study however, found a significantly better outcome in all above mentioned perioperative parameters, which reinforces the importance of the applied surgical technique. Moreover, lack of standardization in off-clamp surgical approach requires more comprehensive studies in order to establish proper technique with adequate hemostasis and preservation of surrounding parenchyma (15). This concept is strongly emphasized by Maurice MJ et al. (16), where volume loss of renal parenchyma was recognized to be the most important modifiable determinant of long term renal function. Tissue sparing technique with simple hemostatic principles could assist in achieving this goal. Kreigmar MC et al. (17) identified 40 cases of open partial nephrectomies, performed without clamping of the renal artery. The mean operative time was shorter compared to our study (106 vs $162.5 \mathrm{~min}$ ), but EBL was significantly higher (521 vs $250 \mathrm{ml}$ ); nevertheless, their study included patients with PADUA scores $>8$ and more complexity of tumour localization.
This indicates that our surgical technique could be improved, since more favourable PADUA scores and tumour positions should lead to shorter operative time. However, complication rate and surgical margin status were comparable between studies.

The most common tumour location in our study was upper pole and the majority of patients were elective, with a normal contralateral kidney. This is an important prerequisite for normal postoperative kidney function. Serum creatinine is the easiest and most commonly used tool to assess RF after PN; however, it is not reliable since its value is significantly affected by age, sex, and muscle mass, especially in the presence of a healthy contralateral kidney (18). Nevertheless, determination of GFR has been shown to reflect RF more accurately than serum creatinine. Our study showed significant improvement in RF in the early postoperative period after using RSHT, comparing GFR values between groups. This confirms effectiveness of our technique, even in patients with solitary or bilateral kidney tumours.

The impact of different resection and renorrhaphy techniques on postoperative RF and perioperative blood loss has not been sufficiently investigated, and standardized reporting of these techniques for future PN series is warranted (19). Our technique consisted of nephron sparing PN with running suture of the surgical edges and fat tissue tamponade of the surgical bed and showed promising results. Operative time and EBL were at least comparable to other studies using open or minimal invasive ZTPN, with preservation of renal function, negative surgical margins and no signs of tumour recurrence during a median of three years follow up time, with additional low transfusion rate and relatively short hospital stay. Our research could be a starting point for future, so that more comprehensive studies comparing different surgical approaches could be developed.

This research, however, has its limitations. First of all, our study concerned only patients treated with an open approach, a single technique and performed by one surgeon. Additionally, our study included only one patient with T2 stage RCC with a relatively low PADUA score. Only few patients had a solitary kidney tumour, so postoperative RF could not be accurately estimated without renal scintigraphy. Finally, the majority of tumours had polar localization (84.4\%) and exophitic growth (78.9\%), therefore, the risk of complications in our study population may have been inherently lower.

During PN surgery, the most important considerations in preserving RF are efforts at minimizing blood loss and reducing operative time, while maximizing renal parenchyma volume. Our surgical technique showed satisfactory results regarding all perioperative outcomes, with no additional technical requirements. It could be safely performed in local, low volume facilities, thus reducing the need for expensive and more challenging minimal invasive surgical techniques.

\section{Statement of ethics}

Each subject signed the acceptance of the study protocol, in which the Ethical Principles for Medical Research Involving Human Subjects (The Helsinki Declaration) were clearly stated. They all signed the written consent form. 
The National Ethical Committee at the University of Montenegro approved this study.

\section{References}

1. Peycelon M, Hupertan V, Comperat E, et al. Long-Term Outcomes After Nephron Sparing Surgery for Renal Cell Carcinoma Larger than $4 \mathrm{~cm}$. J Urol. 2009; 181:35-41.

2. Diblasio CJ, Snyder ME, Russo P. Mini-flank supra 11-th rib incision for open partial or radical nephrectomy. BJU Int. 2006; 97:149-56.

3. Wang H, Sun LA, Wang Y, et al. Mini-flank supra-12th rib incision for open partial nephrectomy for renal tumor with RENAL nephrometry score $\geq 10$ : an innovation of traditional open surgery. Medicine. 2015; 94:692.

4. Browne C, Lonergan PE, Bolton EM, et al. A single centre experience of zero-ischaemia laparoscopic partial nephrectomy in Ireland. Ir J Med Sci. 2017; 186:1023-1026.

5. Rod X, Peyronnet B, Seisen T, et al. Impact of ischaemia time on renal function after partial nephrectomy: a systematic review. BJU int. 2016; 118:692-705.

6. Simone G, Gill IS, Mottrie A, et al. Indications, techniques, outcomes and limitations for minimally ischemic and off-clamp partial nephrectomy: a systematic review of the literature. Eur Urol. 2015; 68:632-40.

7. Lopez-Beltran A, Scarpelli M, Montironi R, et al. 2004 WHO classification of the renal tumors of the adults. Eur Urol. 2006; 49:798-805.

8. Dindo D, Demartines N, Clavien PA. Classification of surgical complications: a new proposal with evaluation in a cohort of 6336 patients and results of a survey. Ann Surg. 2004; 240:205-13.

9. Lopez-Beltran A, Carrasco JC, Cheng L, et al. 2009 update on the classification of renal epithelial tumors in adults. Int J Urol. 2009; 16:432-43.

10. Russo P, Mano R, Kimm S. Open Partial Nephrectomy. In: Kean $T E$ and Graham SD, editors. Glenn's Urologic surgery. Lippincott Williams \& Wilkins, Wolters Kluwer; 2016, p. 7-16.

11. Gill IS, Patil MB, Abreu AL, et al. Zero ischemia anatomical partial nephrectomy: a novel approach. J Urol. 2012; 187:807-15.

12. Minervini A, Ficarra $V$, Rocco F, et al. Simple enucleation is equivalentto traditional partial nephrectomy for renal cell carcinoma: results of a nonrandomized, retrospective, comparative study. J Urol. 2011; 185:1604-10.

13. Desai M, de Castro Abreu AL, Leslie S, et al. Robotic partial nephrectomy with superselective versus main artery clamping: a retrospective comparison. Eur Urol. 2014; 66:713-9.

14. Smith GL, Kenney PA, Lee Y, et al. Non-clamped partial nephrectomy: techniques and surgical outcomes. BJU Int. 2011; 107:1054-8.

15. Kopp RP, Mehrazin R, Palazzi K, et al. Factors affecting renal function after open partial nephrectomy - a comparison of clampless and clamped warm ischemic technique. Urology. 2012; 80:865-70.

16. Maurice MJ, Ramirez D, Maloc E, et al. Predictors of excisional volume loss in partial nephrectomy: is there still room for improvement? Eur Urol. 2016; 70:413-5.

17. Kriegmar M.C, Pfalzgraf D, Hacker A, et al. ZIRK -Technique: Zero ischemia resection in the kidney for high-risk renal masses: Perioperative outcome. Urol Int. 2015; 95:216-222.

18. Volpe A, Blute ML, Ficarra V, et al. Renal Ischemia and Function After Partial Nephrectomy: A Collaborative Review of the Literature. Eur Urol. 2015; 68:61-74.

19. Minervini A, Carini M, Uzzo RG, et al. Standardized reporting of resection technique during nephron sparing surgery: the surfaceintermediate-base margin score. Eur Urol. 2014; 66:803-5.

\author{
Correspondence \\ Petar Kavaric, MD, PhD \\ petar.kavaric@kccg.me \\ Aleksandar Magdelinic, MD \\ acomgd@yahoo.com \\ Marko Vukovic, MD (Corresponding Author) \\ marko.vukovic09@gmail.com \\ Department of Urology, Clinical centre of Montenegro \\ Ljubljanska bb, 81000 Podgorica, Montenegro
}

